\title{
The Analysis of the Dispersion Characteristic of Impact Point Sequence Coordinates with Armament Projecting on Fixed Targets
}

\author{
Xiangwen Leng, Xu Zhang, Bo Li and Lin Shao \\ Dalian Naval Academy, Liaoning China
}

\begin{abstract}
-when armament projecting on fixed target is conducted, the deviation of the actual position of the impact point relative to the predetermined position has substantial randomness, causing difficulty in the analysis of armament scale or concentrated application effect. By building sequence coordinates for impact point coordinate components according to certain rules, with the distribution theory of multiple random variables(probability theory), obtain the regularity that the expected sequence coordinates of individual impact points "on the straight line which can be expressed by math"; meanwhile, put forward a simple and convenient method of calculating the average and variance of impact point sequence coordinates of individual through key coefficients and lay foundation for making researches on the evaluation of armament application efficiency and optimization of firing parameters.
\end{abstract}

Keywords-impact point; sequence; dispersion; coefficient

\section{INTRODUCTION}

Generally, in the process that the armament launched from the platform is projected on fixed target by the motive power, the deviation of the actual position of the impact point relative to the predetermined position has substantial randomness due to the fact that the armament can arrive at the target position after flying or navigating in long distance, for instance, the shells that are fired and the self-propelled mines that are laid. Under the influence of such factors like the errors in flight or navigation, platform position errors, etc., the randomness is not only related to the situation that whether the individual armament could hit the target or not or enter the predetermined area, but also influence the effect that multiple armaments fire on fixed targets scale or intensive application. In this circumstance, the analysis of dispersion characteristic of impact points is the basis of making researches on application efficiency evaluation and firing parameter optimization, and the regularity of impact point sequence dispersion is one of the basic ways to solve the above-mentioned problems.

\section{THE DEFINITION AND DISPERSION REGULARITY OF IMPACT POINT SEQUENCE COORDINATES}

\section{A. The Definition of Impact Point Sequence Coordinates}

Supposing armaments are projecting on fixed targets and $n$ impact points will be formed. Under the influence of various errors, No. j impact point coordinate $\left(X_{j}, Y_{j}\right)_{(j=\overline{1, n})}$ comply with two-dimensional normal distribution, and the corresponding distribution parameters are $\bar{x}_{j}, \bar{y}_{j}, \sigma_{x_{j}}, \sigma_{y_{j}}$, $r_{x_{j} y_{j}}$. Arrange the $\mathrm{X}$ components of $\mathrm{n}$ impact point coordinates in the sequence from small to large, $X_{(1)} \leq X_{(2)} \leq \cdots \leq X_{(n)}$. Here $X_{(s)}(s=\overline{1, n})$ is called impact point horizontal sequence coordinate and the corresponding impact point vertical coordinate is named as $Y_{[s]}$ and is called impact point vertical adjoin sequence coordinate. the 2dimensional random variable $\left(X_{(s)}, Y_{[s]}\right)$ is called impact point sequence coordinate.

\section{B. The Dispersion Regularity of Impact Point Sequence Coordinates}

According to the definition of impact point sequence coordinates and the relevant knowledge of probability theory, the distribution density function[1,2] is as follows,

$$
f_{x_{(s)}, y_{[s]}}(x, y)=\sum_{j=1}^{n} \Psi_{s, j}(x) f_{x_{j} y_{j}}(x, y) \quad(s=\overline{1, n})
$$

Hereinto, the mathematical expression of auxiliary function $\Psi_{s, j}(x)$ is as follows,

$$
\begin{gathered}
\Psi_{1, j}(x)=\prod_{\substack{k=1 \\
k \neq j}}^{n}\left[1-F_{x_{k}}(x)\right] \Psi_{n, j}(x)=\prod_{\substack{k=1 \\
k \neq j}}^{n} F_{x_{k}}(x) \\
\Psi_{s, j}(x)=\sum_{\substack{r_{1}=1 \\
r_{1} \neq j}}^{n-s+2} \sum_{r_{2}=r_{1}+1}^{n-s+3} \cdots \sum_{\substack{r_{2} \neq j \\
r_{s-1} \neq r_{s-2}+1}}^{n}\left\{\left[\prod_{\substack{k=1 \\
k \neq j}}^{s-1}\left[F_{x_{r k}}(x)\right]\right] \prod_{\substack{l=1 \\
l \neq j, r_{1}, r_{2}, \cdots, r_{s-1}}}^{n}\left[1-F_{x_{l}}(x)\right]\right\} \\
\left(s=\frac{2, n-1 ; j}{1, n}\right)
\end{gathered}
$$

Do the integral to variable $y$ in Formula (1) and obtain the distribution density function of impact point horizontal sequence coordinate $X_{(s)}$

$$
f_{x_{(s)}}(x)=\sum_{j=1}^{n} \Psi_{s, j}(x) f_{x_{j}}(x) \quad(s=1,2, \cdots, n)
$$

So the average and variance [3 5] of $X_{(s)}$ can be calculated by the following formulas 


$$
\begin{gathered}
\bar{x}_{(s)}=\sum_{j=1}^{n} a_{s, j}^{(1)} \\
D_{x_{(s)}}=\sum_{j=1}^{n} a_{s, j}^{(2)}-(\bar{x}(s))^{2} \quad(s=\overline{1, n})
\end{gathered}
$$

In addition,

$$
a_{s, j}^{(k)}=\int_{-\infty}^{\infty} x^{k} \Psi_{s, j}(x) f_{x_{j}}(x) d x \quad(j, s=\overline{1, n} ; k=0,1, \cdots)
$$

Similarly, the distribution density function of impact point vertical adjoint sequence coordinate $Y_{[s]}$ can be obtained.

$$
f_{y_{[]]}}(y)=\sum_{j=1}^{n} \int_{-\infty}^{\infty} \Psi_{s, j}(x) f_{x_{j} y_{j}}(x, y) d x \quad(s=\overline{1, n})
$$

Then the average and variance of $Y_{[s]}$ are respectively as follows,

$$
\begin{aligned}
\bar{y}_{[s]}=\sum_{j=1}^{n}\left[\bar{y}_{j} a_{s, j}^{(0)}+r_{x_{j} y_{j}} \frac{\sigma_{y_{j}}}{\sigma_{x_{j}}}\left(a_{s, j}^{(1)}-\bar{x}_{j} a_{s, j}^{(0)}\right)\right] \\
D_{y_{[s]}=} \sum_{j=1}^{n}\left\{\left[\bar{y}_{j}^{2}+D_{y_{j}}\left(1-r_{x_{j} y_{j}}^{2}\right)\right] a_{s, j}^{(0)}+2 \bar{y}_{j} \frac{k_{x_{j} y_{j}}}{D_{x_{j}}}\left(a_{s, j}^{(1)}-\bar{x}_{j} a_{s, j}^{(0)}\right)+\right. \\
\\
\left.\frac{k_{x_{j} y_{j}}^{2}}{D_{x_{j}}^{2}}\left(a_{s, j}^{(2)}-2 \bar{x}_{j} a_{s, j}^{(1)}+\bar{x}_{j}^{2} a_{s, j}^{(0)}\right)\right\}-\left(\bar{y}_{[s]}\right)^{2} \quad(s=\overline{1, n})
\end{aligned}
$$

\section{THE DISPERSION CHARACTERISTIC OF IMPACT POINT SEQUENCE COORDINATES WITH ARMAMENT PROJECTING ON FIXED TARGETS}

When armaments are projecting on fixed targets, $\mathrm{n}$ impact points will be formed. Generally it is considered that the dispersion of impact points $\left(X_{j}, Y_{j}\right)$ comply with normal distribution of the same parameters. Supposing the corresponding distribution parameters are respectively as follows,

$$
\begin{aligned}
& \bar{x}_{j}=\bar{x}, \bar{y}_{j}=\bar{y} ; \sigma_{x_{j}}=\sigma_{x}, \sigma_{y_{j}}=\sigma_{y} ; k_{x_{j} y_{j}}=k_{x y} \quad(j=\overline{1, n}) \\
& \text { Supposing } \frac{x-\bar{x}}{\sigma_{x}}=t
\end{aligned}
$$

and then $f_{(s)}(t)=\frac{n}{\sqrt{2 \pi}} C_{n-1}^{s-1} e^{-\frac{t^{2}}{2}}\left[F_{0}(t)\right]^{s-1}\left[1-F_{0}(t)\right]^{n-s}$
Now, the average and variance of impact point horizontal sequence coordinate $X_{(s)}$ are as follows,

$$
\bar{x}_{(s)}=\bar{x}+\sigma_{x} c_{s}
$$

$$
D_{x_{(s)}}=D_{x} d_{s} \quad(s=\overline{1, n})
$$

In the formula, $c_{s}=M\left(T_{s}\right)=\int_{-\infty}^{\infty} t f_{(s)}(t) d t$

$$
d_{s}=D\left(T_{s}\right)=\int_{-\infty}^{\infty} t^{2} f_{(s)}(t) d t-c_{s}^{2}
$$

The average and variance of impact point vertical adjoint sequence coordinate $Y_{[s]}$ are as follows,

$$
\bar{y}_{[s]}=\bar{y}+r_{x y} \sigma_{y} c_{s}
$$

$$
D_{y_{[s]}}=D_{y}\left[1-\left(1-d_{s}\right) r_{x y}^{2}\right] \quad(s=\overline{1, n})
$$

Integrate Formulas (9) and (10), under the condition that $n$ impact point coordinates $\left(X_{j}, Y_{j}\right) \quad(j=\overline{1, n})$ comply with the normal distribution when average, variance and covariance are equal, the impact point expectation sequence coordinates $\left(\bar{x}_{(s)}, \bar{y}_{[s]}\right)(s=\overline{1, n})$ are on the same straight line, as follows,

$$
y-\bar{y}=r_{x y} \frac{\sigma_{y}}{\sigma_{x}}(x-\bar{x})
$$

\section{THE SIMPLIFIED CALCULATION OF THE DISPERSION OF THE IMPACT POINT SEQUENCE COORDINATES}

To sum up, in the condition of armaments projecting on fixed targets, the average and variance of the impact point sequence coordinates are not only dependent on their distribution parameters, but also related to coefficients $c_{s}$ and $d_{s}$ which have the following characteristics:

$$
c_{n+1-s}=-c_{s} \quad d_{n+1-s}=d_{s} \quad(s=\overline{1, n})
$$

Therefore, when $n$ is certain, what is required is only to provide the corresponding $c_{s}$ and $d_{s}$ in the table when $s \leq \operatorname{Int}\left(\frac{n}{2}\right)$. Table 1 provides the the corresponding $c_{s}$ and $d_{s}$ values under certain circumstances. 
TABLE I. PART OF THE COEFFICIENTS $c_{s}$ AND $d_{s}$ VALUES

\begin{tabular}{|c|c|c|c|c|c|c|c|c|c|}
\hline$n$ & 2 & 3 & \multicolumn{2}{|c|}{4} & \multicolumn{2}{|c|}{5} & \multicolumn{3}{|c|}{6} \\
\hline$S$ & 1 & 1 & 1 & 2 & 1 & 2 & 1 & 2 & 3 \\
\hline$-c_{s}$ & 0.5662 & 0.8483 & 1.0314 & 0.2990 & 1.1650 & 0.4970 & 1.2692 & 0.6438 & 0.2036 \\
\hline$d_{s}$ & 0.7559 & 1.3690 & 1.9696 & 0.6136 & 2.5696 & 1.2142 & 3.1696 & 1.8143 & 0.6007 \\
\hline
\end{tabular}

Obviously, in the condition that the parameters $n$ and $s$ are given, we can find the corresponding constant $c_{s}$, and conveniently make use of Formula (9)and(10) to calculate the average and variance of horizontal sequence coordinates and vertical adjoins sequence coordinates.

\section{CONCLUSION}

To sum up, when armaments are projecting on fixed targets, and $n$ impact point sequence coordinates comply with normal distribution when average, variance and covariance are equal. The impact point expectation sequence coordinates are on the same straight line; meanwhile, the critical coefficients $c_{s}$ and $d_{s}$ that influence the dispersion of impact point sequence coordinates are put forth. I n addition, because the critical coefficients are irrelevant to the impact point distribution parameters and only relevant to the armament projection quantity as well as the sequence number of impact points, therefore, we can make numerical tables of these constants under common circumstances, and the personnel concerned can refer to the tables for the corresponding constant values directly according to relevant parameters, or obtain calculation results by computer programming methods so as to conduct tactical calculations.

\section{REFERENCES}

[1] Sun Shanze, Nonparametric Statistic [M], Peking University Press,2000.

[2] Fan Jincheng, Wu Kefa[M], Science Press, 2001.

[3] Zhang Xu, Leng Xiang wen, The Distributed Characteristics of Ordering Coordinates of SLMM's position[J], Fire Control \& Command Control, 2004, 29(9): 47-51. (in Chinese)

[4] Jia Y, Song B W, Li Q W, Mathematical Model and Simulation of Torpedo Weapon System Effectiveness Evaluation[J] Acta Armament, 2006, 27(3): 475-478. (in Chinese)

[5] Zhu Y M, Song E B, Zhou J, et al Optimal dimensionality reduction of sensor data in multi-sensor estimation fusion[J], IEEE Transactions on Signal Processing, 2005, 53(5): 1631-1639. 